\title{
On the Fourth Power Moment of Fourier Coefficients of Cusp Form
}

\author{
Jinjiang Li* \& Panwang Wang ${ }^{\dagger} \quad \& \quad$ Min Zhang ${ }^{\ddagger}$ \\ Department of Mathematics, China University of Mining and Technology*†‡ \\ Beijing 100083, P. R. China
}

\begin{abstract}
Let $a(n)$ be the Fourier coefficients of a holomorphic cusp form of weight $\kappa=2 n \geqslant 12$ for the full modular group and $A(x)=\sum_{n \leqslant x} a(n)$. In this paper, we establish an asymptotic formula of the fourth power moment of $A(x)$ and prove that

$$
\int_{1}^{T} A^{4}(x) \mathrm{d} x=\frac{3}{64 \kappa \pi^{4}} s_{4 ; 2}(\tilde{a}) T^{2 \kappa}+O\left(T^{2 \kappa-\delta_{4}+\varepsilon}\right)
$$

with $\delta_{4}=1 / 8$, which improves the previous result.

Keywords: Cusp form; Fourier coefficient; mean value; asymptotic formula

Mathematics Subject Classification 2010: 11N37,11M06
\end{abstract}

\section{Introduction and main result}

Let $a(n)$ be the Fourier coefficients of a holomorphic cusp form of weight $\kappa=2 n \geqslant 12$ for the full modular group. In 1974, Deligne [2] proved the following profound result

$$
a(n) \ll n^{(\kappa-1) / 2} d(n),
$$

where $d(n)$ denotes the Dirichlet divisor function and the implied constant in $\ll$ is absolute. Suppose $x \geqslant 2$ and define

$$
A(x):=\sum_{n \leqslant x} a(n)
$$

It is well known that $A(x)$ has no main term and $A(x) \ll x^{\kappa / 2-1 / 6+\varepsilon}$. In 1973, Joris [5] proved that

$$
A(x)=\Omega_{ \pm}\left(x^{\kappa / 2-1 / 4} \log \log \log x\right) .
$$

\footnotetext{
${ }^{\ddagger}$ Corresponding author.

E-mail addresses: jinjiang.li.math@gmail.com (J. Li), panwangw@gmail.com (P. Wang), min.zhang.math@gmail.com (M. Zhang).
} 
In 1990, Ivić [3] showed that there exist two points $t_{1}$ and $t_{2}$ in the interval $[T, T+$ $\left.C T^{1 / 2}\right]$ such that

$$
A\left(t_{1}\right)>B t_{1}^{\kappa / 2-1 / 4}, \quad A\left(t_{2}\right)<-B t_{2}^{\kappa / 2-1 / 4},
$$

where $B>0, C>0$ are constants. It is conjectured that

$$
A(x) \ll x^{(\kappa-1) / 2+1 / 4+\varepsilon}
$$

is true for every $\varepsilon$. The evidence in support of this conjecture has been given by Ivić [3], who proved the following square mean value formula of $A(x)$, i.e.

$$
\int_{1}^{T} A^{2}(x) \mathrm{d} x=\mathcal{C}_{2} T^{\kappa+1 / 2}+B(T),
$$

where

$$
\begin{gathered}
\mathcal{C}_{2}=\frac{1}{(4 \kappa+2) \pi^{2}} \sum_{n=1}^{\infty} a^{2}(n) n^{-\kappa-1 / 2}, \\
B(T) \ll T^{\kappa} \log ^{5} T, \quad B(T)=\Omega\left(T^{\kappa-1 / 4} \frac{(\log \log \log T)^{3}}{\log T}\right) .
\end{gathered}
$$

In [3], Ivić also proved the upper bound of eighth power moment of $A(x)$, that is

$$
\int_{1}^{T} A^{8}(x) \mathrm{d} x \ll T^{4 \kappa-1+\varepsilon} .
$$

Cai [1] studied the third and fourth power moments of $A(x)$. He proved that

$$
\begin{gathered}
\int_{1}^{T} A^{3}(x) \mathrm{d} x=\mathcal{C}_{3} T^{(6 \kappa+1) / 4}+O\left(T^{(6 \kappa+1) / 4-\delta_{3}+\varepsilon}\right), \\
\int_{1}^{T} A^{4}(x) \mathrm{d} x=\mathcal{C}_{4} T^{2 \kappa}+O\left(T^{2 \kappa-\delta_{4}+\varepsilon}\right),
\end{gathered}
$$

where $\delta_{3}=1 / 14, \delta_{4}=1 / 23$ and

$$
\begin{gathered}
\mathcal{C}_{3}:=\frac{3}{4(6 \kappa+1) \pi^{3}} \sum_{\substack{n, m, k \in \mathbb{N} \\
\sqrt{n}+\sqrt{m}=\sqrt{k}}}(n m k)^{-\kappa / 2-1 / 4} a(n) a(m) a(k), \\
\mathcal{C}_{4}:=\frac{3}{64 \kappa \pi^{4}} \sum_{\substack{n, m, k, \ell \in \mathbb{N} \\
\sqrt{n}+\sqrt{m}=\sqrt{k}+\sqrt{\ell}}}(n m k \ell)^{-\kappa / 2-1 / 4} a(n) a(m) a(k) a(\ell) .
\end{gathered}
$$

In [10], Zhai proved that (1.3) holds for $\delta_{3}=1 / 4$. Following the approach of Tsang [9], Zhai [10] proved that the equation (1.4) holds for $\delta_{4}=2 / 41$. This approach used the method of exponential sums. In particular, if the exponent pair conjecture is true, namely, if $(\varepsilon, 1 / 2+\varepsilon)$ is an exponent pair, then the equation (1.4) holds for $\delta_{4}=1 / 14$. 
Later, combining the method of [4] and a deep result of Robert and Sargos [8], Zhai [12] proved that the equation (1.4) holds for $\delta_{4}=3 / 28$. By a unified approach, Zhai [11] proved that the asymptotic formula

$$
\int_{1}^{T} A^{k}(x) \mathrm{d} x=\mathcal{C}_{k} T^{1+k(2 \kappa-1) / 4}+O\left(T^{1+k(2 \kappa-1) / 4-\delta_{k}+\varepsilon}\right)
$$

holds for $3 \leqslant k \leqslant 7$, where $\mathcal{C}_{k}$ and $0<\delta_{k}<1$ are explicit constants.

The aim of this paper is to improve the value of $\delta_{4}=3 / 28$, which is achieved by Zhai [12]. The main result is the following

Theorem 1.1 We have

$$
\int_{1}^{T} A^{4}(x) \mathrm{d} x=\frac{3}{64 \kappa \pi^{4}} s_{4 ; 2}(\tilde{a}) T^{2 \kappa}+O\left(T^{2 \kappa-\delta_{4}+\varepsilon}\right)
$$

with $\delta_{4}=1 / 8$, where

$$
s_{4 ; 2}(\tilde{a})=\sum_{\substack{n, m, k, \ell \in \mathbb{N}^{*} \\ \sqrt{n}+\sqrt{m}=\sqrt{k}+\sqrt{\ell}}} \frac{a(n) a(m) a(k) a(\ell)}{(n m k \ell)^{\kappa / 2+1 / 4}} .
$$

Notation. Throughout this paper, $a(n)$ be the Fourier coefficients of a holomorphic cusp form of weight $\kappa=2 n \geqslant 12$ for the full modular group; $d(n)$ denote the Dirichlet divisor function; $\tilde{a}(n):=a(n) n^{-\kappa / 2+1 / 2} ;\|x\|$ denotes the distance from $x$ to the nearest integer, i.e., $\|x\|=\min _{n \in \mathbb{Z}}|x-n| .[x]$ denotes the integer part of $x ; n \sim N$ means $N<n \leqslant$ $2 N ; n \asymp N$ means $C_{1} N \leqslant n \leqslant C_{2} N$ with positive constants $C_{1}, C_{2}$ satisfying $C_{1}<C_{2}$. $\varepsilon$ always denotes an arbitrary small positive constant which may not be the same at different occurances. We shall use the estimates $d(n) \ll n^{\varepsilon}$. Suppose $f: \mathbb{N} \rightarrow \mathbb{R}$ is any function satisfying $f(n) \ll n^{\varepsilon}, k \geqslant 2$ is a fixed integer. Define

$$
s_{k ; \ell}(f):=\sum_{\substack{n_{1}, \cdots, n_{\ell}, n_{\ell+1}, \cdots, n_{k} \in \mathbb{N}^{*} \\ \sqrt{n_{1}}+\cdots+\sqrt{n_{l}}=\sqrt{n_{\ell+1}}+\cdots+\sqrt{n_{k}}}} \frac{f\left(n_{1}\right) f\left(n_{2}\right) \cdots f\left(n_{k}\right)}{\left(n_{1} n_{2} \cdots n_{k}\right)^{3 / 4}}, \quad 1 \leqslant \ell<k .
$$

We shall use $s_{k ; \ell}(f)$ to denote both of the series (1.5) and its value. Suppose $y>1$ is a large parameter, and we define

$$
s_{k ; \ell}(f ; y):=\sum_{\substack{n_{1}, \cdots, n_{\ell}, n_{\ell+1}, \cdots, n_{k} \leqslant y \\ \sqrt{n_{1}}+\cdots+\sqrt{n_{l}}=\sqrt{n_{\ell+1}}+\cdots+\sqrt{n_{k}}}} \frac{f\left(n_{1}\right) f\left(n_{2}\right) \cdots f\left(n_{k}\right)}{\left(n_{1} n_{2} \cdots n_{k}\right)^{3 / 4}}, \quad 1 \leqslant \ell<k .
$$

\section{Preliminary Lemmas}

Lemma 2.1 If $g(x)$ and $h(x)$ are continuous real-valued functions of $x$ and $g(x)$ is monotonic, then

$$
\int_{a}^{b} g(x) h(x) \mathrm{d} x \ll\left(\max _{a \leqslant x \leqslant b}|g(x)|\right)\left(\max _{a \leqslant u<v \leqslant b}\left|\int_{u}^{v} h(x) \mathrm{d} x\right|\right) .
$$


Proof. See Tsang [9], Lemma 1.

Lemma 2.2 Suppose $A, B \in \mathbb{R}, A \neq 0$. . Then we have

$$
\int_{T}^{2 T} t^{\alpha} \cos (A \sqrt{t}+B) \mathrm{d} t \ll T^{1 / 2+\alpha}|A|^{-1}
$$

Proof. It follows from Lemma 2.1 easily.

Lemma 2.3 If $n, m, k, \ell \in \mathbb{N}$ such that $\sqrt{n}+\sqrt{m} \pm \sqrt{k}-\sqrt{\ell} \neq 0$, then there hold

$$
|\sqrt{n}+\sqrt{m} \pm \sqrt{k}-\sqrt{\ell}| \gg(n m k \ell)^{-1 / 2} \max (n, m, k, \ell)^{-3 / 2},
$$

respectively.

Proof. See Kong [7], Lemma 3.2.1.

Lemma 2.4 Let $f: \mathbb{N} \rightarrow \mathbb{R}$ be any function satisfying $f(n) \ll n^{\varepsilon}$. Then we have

$$
\left|s_{k ; \ell}(f)-s_{k ; \ell}(f ; y)\right| \ll y^{-1 / 2+\varepsilon}, \quad 1 \leqslant \ell<k,
$$

where $k \geqslant 2$ is a fixed integer.

Proof. See Zhai [11], Lemma 3.1.

Lemma 2.5 Suppose $1 \leqslant N \leqslant M, 1 \leqslant K \leqslant L, N \leqslant K, M \asymp L, 0<\Delta \ll L^{1 / 2}$. Let $\mathscr{A}_{1}(N, M, K, L ; \Delta)$ denote the number of solutions of the following inequality

$$
0<|\sqrt{n}+\sqrt{m}-\sqrt{k}-\sqrt{\ell}|<\Delta
$$

with $n \sim N, m \sim M, k \sim K, \ell \sim L$. Then we have

$$
\mathscr{A}_{1}(N, M, K, L ; \Delta) \ll \Delta L^{1 / 2} N M K+N K L^{1 / 2+\varepsilon} .
$$

Especially, if $\Delta L^{1 / 2} \gg 1$, then

$$
\mathscr{A}_{1}(N, M, K, L ; \Delta) \ll \Delta L^{1 / 2} N M K .
$$

Proof. See Zhai [12], Lemma 5.

Lemma 2.6 Suppose $N_{j} \geqslant 2(j=1,2,3,4), \Delta>0$. Let $\mathscr{A}_{ \pm}\left(N_{1}, N_{2}, N_{3}, N_{4} ; \Delta\right)$ denote the number of solutions of the following inequality

$$
0<\left|\sqrt{n_{1}}+\sqrt{n_{2}} \pm \sqrt{n_{3}}-\sqrt{n_{4}}\right|<\Delta
$$

with $n_{j} \sim N_{j}(j=1,2,3,4), n_{j} \in \mathbb{N}^{*}$. Then we have

$$
\mathscr{A}_{ \pm}\left(N_{1}, N_{2}, N_{3}, N_{4} ; \Delta\right) \ll \prod_{j=1}^{4}\left(\Delta^{1 / 4} N_{j}^{7 / 8}+N_{j}^{1 / 2}\right) N_{j}^{\varepsilon} .
$$

Proof. See Zhai [12], Lemma 3. 


\section{Proof of Theorem 1.1}

In this section, we shall prove the theorem. We begin with the following truncated formula, which is proved by Jutila [6], i.e.,

$$
A(x)=\frac{1}{\sqrt{2} \pi} \sum_{n \leqslant N} \frac{a(n)}{n^{\kappa / 2+1 / 4}} x^{\kappa / 2-1 / 4} \cos (4 \pi \sqrt{n x}-\pi / 4)+O\left(x^{\kappa / 2+\varepsilon} N^{-1 / 2}\right),
$$

where $1 \leqslant N \ll x$.

Suppose $T \geqslant 10$. By a splitting argument, it is sufficient to prove the result in the interval $[T, 2 T]$. Take $y=T^{3 / 4}$. For any $T \leqslant x \leqslant 2 T$, by the truncated formula (3.1), we get

$$
A(x)=\frac{1}{\sqrt{2} \pi} \mathcal{R}(x)+O\left(x^{\kappa / 2+\varepsilon} y^{-1 / 2}\right)
$$

where

$$
\mathcal{R}(x):=x^{\kappa / 2-1 / 4} \sum_{n \leqslant y} \frac{a(n)}{n^{\kappa / 2+1 / 4}} \cos (4 \pi \sqrt{n x}-\pi / 4) .
$$

We have

$$
\begin{aligned}
\int_{T}^{2 T} A^{4}(x) \mathrm{d} x & =\frac{1}{4 \pi^{4}} \int_{T}^{2 T} \mathcal{R}^{4}(x) \mathrm{d} x+O\left(T^{2 \kappa+1 / 4+\varepsilon} y^{-1 / 2}+T^{2 \kappa+1+\varepsilon} y^{-2}\right) \\
& =\frac{1}{4 \pi^{4}} \int_{T}^{2 T} \mathcal{R}^{4}(x) \mathrm{d} x+O\left(T^{2 \kappa-1 / 8+\varepsilon}\right) .
\end{aligned}
$$

Let

$$
g=g(n, m, k, \ell):=\left\{\begin{array}{cc}
\frac{a(n) a(m) a(k) a(\ell)}{(n m k \ell)^{\kappa / 2+1 / 4}}, & \text { if } n, m, k, \ell \leqslant y \\
0, & \text { otherwise. }
\end{array}\right.
$$

According to the elementary formula

$$
\cos a_{1} \cos a_{2} \cdots \cos a_{h}=\frac{1}{2^{h-1}} \sum_{\left(i_{1}, i_{2} \cdots, i_{h-1}\right) \in\{0,1\}^{h-1}} \cos \left(a_{1}+(-1)^{i_{1}} a_{2}+\cdots+(-1)^{i_{h-1}} a_{h}\right),
$$

we can write

$$
\mathcal{R}^{4}(x)=S_{1}(x)+S_{2}(x)+S_{3}(x)+S_{4}(x),
$$

where

$$
\begin{aligned}
S_{1}(x) & :=\frac{3}{8} \sum_{\substack{n, m, k, \ell \leqslant y \\
\sqrt{n}+\sqrt{m}=\sqrt{k}+\sqrt{\ell}}} g x^{2 \kappa-1}, \\
S_{2}(x) & :=\frac{3}{8} \sum_{\substack{n, m, k, \ell \leqslant y \\
\sqrt{n}+\sqrt{m} \neq \sqrt{k}+\sqrt{\ell}}} g x^{2 \kappa-1} \cos (4 \pi(\sqrt{n}+\sqrt{m}-\sqrt{k}-\sqrt{\ell}) \sqrt{x}),
\end{aligned}
$$




$$
\begin{aligned}
& S_{3}(x):=\frac{1}{2} \sum_{\substack{n, m, k, \ell \leqslant y \\
\sqrt{n}+\sqrt{m}+\sqrt{k} \neq \sqrt{\ell}}} g x^{2 \kappa-1} \cos \left(4 \pi(\sqrt{n}+\sqrt{m}+\sqrt{k}-\sqrt{\ell}) \sqrt{x}-\frac{\pi}{2}\right), \\
& S_{4}(x):=\frac{1}{8} \sum_{n, m, k, \ell \leqslant y} g x^{2 \kappa-1} \cos (4 \pi(\sqrt{n}+\sqrt{m}+\sqrt{k}+\sqrt{\ell}) \sqrt{x}-\pi) .
\end{aligned}
$$

By (1.1) and Lemma 2.4, we get

$$
\begin{aligned}
\int_{T}^{2 T} S_{1}(x) \mathrm{d} x & =\frac{3}{8} s_{4 ; 2}\left(a(n) n^{-\kappa / 2+1 / 2} ; y\right) \int_{T}^{2 T} x^{2 \kappa-1} \mathrm{~d} x \\
& =\frac{3}{8} s_{4 ; 2}(\tilde{a} ; y) \int_{T}^{2 T} x^{2 \kappa-1} \mathrm{~d} x \\
& =\frac{3}{8} s_{4 ; 2}(\tilde{a}) \int_{T}^{2 T} x^{2 \kappa-1} \mathrm{~d} x+O\left(T^{2 \kappa} y^{-1 / 2+\varepsilon}\right) \\
& =\frac{3}{8} s_{4 ; 2}(\tilde{a}) \int_{T}^{2 T} x^{2 \kappa-1} \mathrm{~d} x+O\left(T^{2 \kappa-3 / 8+\varepsilon}\right) .
\end{aligned}
$$

We now proceed to consider the contribution of $S_{4}(x)$. Applying Lemma 2.2 and (1.1), we get

$$
\begin{aligned}
\int_{T}^{2 T} S_{4}(x) \mathrm{d} x & =\frac{1}{8} \sum_{n, m, k, \ell \leqslant y} g \int_{T}^{2 T} x^{2 \kappa-1} \cos (4 \pi(\sqrt{n}+\sqrt{m}+\sqrt{k}+\sqrt{\ell}) \sqrt{x}-\pi) \mathrm{d} x \\
& \ll \sum_{n, m, k, \ell \leqslant y} \frac{g T^{2 \kappa-1 / 2}}{\sqrt{n}+\sqrt{m}+\sqrt{k}+\sqrt{\ell}} \\
& =T^{2 \kappa-1 / 2} \sum_{n, m, k, \ell \leqslant y} \frac{a(n) a(m) a(k) a(\ell)}{(n m k \ell)^{(\kappa-1) / 2}(n m k \ell)^{3 / 4}} \cdot \frac{1}{\sqrt{n}+\sqrt{m}+\sqrt{k}+\sqrt{\ell}} \\
& \ll T^{2 \kappa-1 / 2} \sum_{n, m, k, \ell \leqslant y} \frac{d(n) d(m) d(k) d(\ell)}{(n m k \ell)^{3 / 4} \ell^{1 / 2}} \\
& \ll T^{2 \kappa-1 / 2+\varepsilon} \sum_{n, m, k, \ell \leqslant y} \frac{1}{(n m k)^{3 / 4} \ell^{5 / 4}} \\
& \ll T^{2 \kappa-1 / 2+\varepsilon} y^{1 / 2} \ll T^{2 \kappa-1 / 8+\varepsilon} .
\end{aligned}
$$

Now let us consider the contribution of $S_{2}(x)$. By the first derivative test. we have

$$
\begin{aligned}
\int_{T}^{2 T} S_{2}(x) \mathrm{d} x & \ll \sum_{\substack{n, m, k, \ell \leqslant y \\
\sqrt{n}+\sqrt{m} \neq \sqrt{k}+\sqrt{\ell}}} g \min \left(T^{2 \kappa}, \frac{T^{2 \kappa-1 / 2}}{|\sqrt{n}+\sqrt{m}-\sqrt{k}-\sqrt{\ell}|}\right) \\
& \ll x^{\varepsilon} \mathcal{G}(N, M, K, L),
\end{aligned}
$$

where

$$
\mathcal{G}(N, M, K, L)=\sum_{\substack{\sqrt{n}+\sqrt{m} \neq \sqrt{k}+\sqrt{\ell} \\ n \sim N, m \sim M, k \sim K, \ell \sim L \\ 1 \leqslant N \leqslant M \leqslant y \\ 1 \leqslant K \leqslant L \leqslant y}} g \cdot \min \left(T^{2 \kappa}, \frac{T^{2 \kappa-1 / 2}}{|\sqrt{n}+\sqrt{m}-\sqrt{k}-\sqrt{\ell}|}\right) .
$$


If $M \geqslant 200 L$, then $|\sqrt{n}+\sqrt{m}-\sqrt{k}-\sqrt{\ell}| \gg M^{1 / 2}$, so the trivial estimate yields

$$
\mathcal{G}(N, M, K, L) \ll \frac{T^{2 \kappa-1 / 2+\varepsilon} N M K L}{(N M K L)^{3 / 4} M^{1 / 2}} \ll T^{2 \kappa-1 / 2+\varepsilon} y^{1 / 2} \ll T^{2 \kappa-1 / 8+\varepsilon} .
$$

If $L \geqslant 200 M$, we can get the same estimate. So later we always suppose that $M \asymp L$. Let $\eta=\sqrt{n}+\sqrt{m}-\sqrt{k}-\sqrt{\ell}$. Write

$$
\mathcal{G}(N, M, K, L)=\mathcal{G}_{1}+\mathcal{G}_{2}+\mathcal{G}_{3},
$$

where

$$
\begin{aligned}
\mathcal{G}_{1} & :=T^{2 \kappa} \sum_{0<|\eta| \leqslant T^{-1 / 2}} g, \\
\mathcal{G}_{2} & :=T^{2 \kappa-1 / 2} \sum_{T^{-1 / 2}<|\eta| \leqslant 1} g|\eta|^{-1}, \\
\mathcal{G}_{3} & :=T^{2 \kappa-1 / 2} \sum_{|\eta|>1} g|\eta|^{-1} .
\end{aligned}
$$

We estimate $\mathcal{G}_{1}$ first. By Lemma 2.5, we get

$$
\begin{aligned}
\mathcal{G}_{1} & \ll \frac{T^{2 \kappa+\varepsilon}}{(N M K L)^{3 / 4}} \mathscr{A}_{1}\left(N, M, K, L ; T^{-1 / 2}\right) \\
& \ll \frac{T^{2 \kappa+\varepsilon}}{(N M K L)^{3 / 4}}\left(T^{-1 / 2} L^{1 / 2} N M K+N K L^{1 / 2}\right) \\
& \ll T^{2 \kappa-1 / 2+\varepsilon}(N K)^{1 / 4}+T^{2 \kappa+\varepsilon}(N K)^{1 / 4} L^{-1} \\
& \ll T^{2 \kappa-1 / 2+\varepsilon} y^{1 / 2}+T^{2 \kappa+\varepsilon}(N K)^{1 / 4} L^{-1} \\
& \ll T^{2 \kappa-1 / 8+\varepsilon}+T^{2 \kappa+\varepsilon}(N K)^{1 / 4} L^{-1} .
\end{aligned}
$$

On the other hand, by Lemma 2.6, without loss of generality, we assume that $N \leqslant$ $K \leqslant L$ and obtain

$$
\begin{aligned}
\mathcal{G}_{1} & \ll \frac{T^{2 \kappa+\varepsilon}}{(N M K L)^{3 / 4}} \mathscr{A}_{-}\left(N, M, K, L ; T^{-1 / 2}\right) \\
& \ll \frac{T^{2 \kappa+\varepsilon}}{(N M K L)^{3 / 4}}\left(T^{-1 / 8} N^{7 / 8}+N^{1 / 2}\right)\left(T^{-1 / 8} K^{7 / 8}+K^{1 / 2}\right)\left(T^{-1 / 4} L^{7 / 4}+L\right) \\
& \ll T^{2 \kappa+\varepsilon}(N K)^{-1 / 4} L^{-1 / 2}\left(T^{-1 / 8} N^{3 / 8}+1\right)\left(T^{-1 / 8} K^{3 / 8}+1\right)\left(T^{-1 / 4} L^{3 / 4}+1\right) \\
& \ll T^{2 \kappa+\varepsilon}(N K)^{-1 / 4} L^{-1 / 2}\left(T^{-1 / 4}(N K)^{3 / 8}+T^{-1 / 8} K^{3 / 8}+1\right)\left(T^{-1 / 4} L^{3 / 4}+1\right) \\
& \ll T^{2 \kappa-1 / 4+\varepsilon}(N K)^{1 / 8} L^{-1 / 2} \\
& +T^{2 \kappa+\varepsilon}(N K)^{-1 / 4} L^{-1 / 2}\left(T^{-1 / 8} K^{3 / 8}+1\right)\left(T^{-1 / 4} L^{3 / 4}+1\right) \\
\ll & T^{2 \kappa-1 / 4+\varepsilon} L^{-1 / 4}+T^{2 \kappa+\varepsilon}(N K)^{-1 / 4} L^{-1 / 2}\left(T^{-3 / 8} L^{9 / 8}+1\right) \\
& \ll T^{2 \kappa-1 / 4+\varepsilon}+T^{2 \kappa+\varepsilon}(N K)^{-1 / 4} L^{-1 / 2}\left(T^{-3 / 8} L^{9 / 8}+1\right) .
\end{aligned}
$$


From (3.9) and (3.10), we get

$$
\mathcal{G}_{1} \ll T^{2 \kappa-1 / 8+\varepsilon}+T^{2 \kappa+\varepsilon} \cdot \min \left(\frac{(N K)^{1 / 4}}{L}, \frac{T^{-3 / 8} L^{9 / 8}+1}{(N K)^{1 / 4} L^{1 / 2}}\right) .
$$

Case 1 If $L \gg T^{1 / 3}$, then $T^{-3 / 8} L^{9 / 8} \gg 1$, we get

$$
\begin{aligned}
\mathcal{G}_{1} & \ll T^{2 \kappa-1 / 8+\varepsilon}+T^{2 \kappa+\varepsilon} \cdot \min \left(\frac{(N K)^{1 / 4}}{L}, \frac{T^{-3 / 8} L^{9 / 8}}{(N K)^{1 / 4} L^{1 / 2}}\right) \\
& \ll T^{2 \kappa-1 / 8+\varepsilon}+T^{2 \kappa+\varepsilon}\left(\frac{(N K)^{1 / 4}}{L}\right)^{1 / 2}\left(\frac{T^{-3 / 8} L^{9 / 8}}{(N K)^{1 / 4} L^{1 / 2}}\right)^{1 / 2} \\
& \ll T^{2 \kappa-1 / 8+\varepsilon}+T^{2 \kappa-3 / 16+\varepsilon} L^{-3 / 16} \ll T^{2 \kappa-1 / 8+\varepsilon} .
\end{aligned}
$$

Case 2 If $L \ll T^{1 / 3}$, then $T^{-3 / 8} L^{9 / 8} \ll 1$. By noting that $M \asymp L \asymp \max (N, M, K, L)$ and Lemma 2.3, we have

$$
T^{-1 / 2} \gg|\eta| \gg(n m k \ell)^{-1 / 2} \max (n, m, k, \ell)^{-3 / 2} \asymp(N K)^{-1 / 2} L^{-5 / 2} .
$$

Hence, we obtain

$$
\begin{aligned}
\mathcal{G}_{1} & \ll T^{2 \kappa-1 / 8+\varepsilon}+T^{2 \kappa+\varepsilon} \min \left(\frac{(N K)^{1 / 4}}{L}, \frac{1}{(N K)^{1 / 4} L^{1 / 2}}\right) \\
& \ll T^{2 \kappa-1 / 8+\varepsilon}+T^{2 \kappa+\varepsilon}\left(\frac{(N K)^{1 / 4}}{L}\right)^{1 / 4}\left(\frac{1}{(N K)^{1 / 4} L^{1 / 2}}\right)^{3 / 4} \\
& =T^{2 \kappa-1 / 8+\varepsilon}+T^{2 \kappa+\varepsilon}(N K)^{-1 / 8} L^{-5 / 8} \\
& \ll T^{2 \kappa-1 / 8+\varepsilon}+T^{2 \kappa+\varepsilon}\left(T^{-1 / 2}\right)^{1 / 4} \ll T^{2 \kappa-1 / 8+\varepsilon} .
\end{aligned}
$$

Combining (3.11) and (3.12), we get

$$
\mathcal{G}_{1} \ll T^{2 \kappa-1 / 8+\varepsilon} \text {. }
$$

Now, we estimate $\mathcal{G}_{2}$. By a splitting argument, we get that there exists some $\delta$ satisfying $T^{-1 / 2} \ll \delta \ll 1$ such that

$$
\mathcal{G}_{2} \ll \frac{T^{2 \kappa-1 / 2+\varepsilon}}{(N M K L)^{3 / 4} \delta} \times \sum_{\substack{\delta<|\eta| \leqslant 2 \delta \\ \eta \neq 0}} 1 .
$$

By Lemma2.5, we get

$$
\begin{aligned}
\mathcal{G}_{2} & \ll \frac{T^{2 \kappa-1 / 2+\varepsilon}}{(N M K L)^{3 / 4} \delta} \mathscr{A}_{1}(N, M, K, L ; 2 \delta) \\
& \ll \frac{T^{2 \kappa-1 / 2+\varepsilon}}{(N M K L)^{3 / 4} \delta}\left(\delta L^{1 / 2} N M K+N K L^{1 / 2}\right) \\
& =T^{2 \kappa-1 / 2+\varepsilon}(N K)^{1 / 4}+T^{2 \kappa-1 / 2+\varepsilon} \delta^{-1}(N K)^{1 / 4} L^{-1} \\
& \ll T^{2 \kappa-1 / 2+\varepsilon} y^{1 / 2}+T^{2 \kappa-1 / 2+\varepsilon} \delta^{-1}(N K)^{1 / 4} L^{-1} \\
& \ll T^{2 \kappa-1 / 8+\varepsilon}+T^{2 \kappa-1 / 2+\varepsilon} \delta^{-1}(N K)^{1 / 4} L^{-1} .
\end{aligned}
$$


On the other hand, by Lemma 2.6, without loss of generality, we assume that $N \leqslant$ $K \leqslant L$ and obtain

$$
\begin{aligned}
\mathcal{G}_{2} & \ll \frac{T^{2 \kappa-1 / 2+\varepsilon}}{(N M K L)^{3 / 4} \delta} \times \mathscr{A}_{-}(N, M, K, L ; 2 \delta) \\
& \ll \frac{T^{2 \kappa-1 / 2+\varepsilon}}{(N M K L)^{3 / 4} \delta}\left(\delta^{1 / 4} N^{7 / 8}+N^{1 / 2}\right)\left(\delta^{1 / 4} K^{7 / 8}+K^{1 / 2}\right)\left(\delta^{1 / 2} L^{7 / 4}+L\right) \\
& \ll T^{2 \kappa-1 / 2+\varepsilon}(N K)^{-1 / 4} L^{-1 / 2} \delta^{-1}\left(\delta^{1 / 4} N^{3 / 8}+1\right)\left(\delta^{1 / 4} K^{3 / 8}+1\right)\left(\delta^{1 / 2} L^{3 / 4}+1\right) \\
& \ll T^{2 \kappa-1 / 2+\varepsilon}(N K)^{-1 / 4} L^{-1 / 2} \delta^{-1}\left(\delta^{1 / 2}(N K)^{3 / 8}+\delta^{1 / 4} K^{3 / 8}+1\right)\left(\delta^{1 / 2} L^{3 / 4}+1\right) \\
\ll & T^{2 \kappa-1 / 2+\varepsilon}(N K)^{1 / 8} L^{-1 / 2} \delta^{-1 / 2} \\
& +T^{2 \kappa-1 / 2+\varepsilon}(N K)^{-1 / 4} L^{-1 / 2} \delta^{-1}\left(\delta^{1 / 4} K^{3 / 8}+1\right)\left(\delta^{1 / 2} L^{3 / 4}+1\right) \\
\ll & T^{2 \kappa-1 / 4+\varepsilon} L^{-1 / 4}+T^{2 \kappa-1 / 2+\varepsilon}(N K)^{-1 / 4} L^{-1 / 2} \delta^{-1}\left(\delta^{3 / 4} L^{9 / 8}+1\right) .
\end{aligned}
$$

From (3.14) and (3.15), we get

$$
\mathcal{G}_{2} \ll T^{2 \kappa-1 / 8+\varepsilon}+T^{2 \kappa-1 / 2+\varepsilon} \delta^{-1} \cdot \min \left(\frac{(N K)^{1 / 4}}{L}, \frac{\delta^{3 / 4} L^{9 / 8}+1}{(N K)^{1 / 4} L^{1 / 2}}\right) .
$$

Case 1 If $\delta \gg L^{-3 / 2}$, then $\delta^{3 / 4} L^{9 / 8} \gg 1$, we get (recall $\delta \gg T^{-1 / 2}$ )

$$
\begin{aligned}
\mathcal{G}_{2} & \ll T^{2 \kappa-1 / 8+\varepsilon}+T^{2 \kappa-1 / 2+\varepsilon} \delta^{-1} \cdot \min \left(\frac{(N K)^{1 / 4}}{L}, \frac{\delta^{3 / 4} L^{9 / 8}}{(N K)^{1 / 4} L^{1 / 2}}\right) \\
& \ll T^{2 \kappa-1 / 8+\varepsilon}+T^{2 \kappa-1 / 2+\varepsilon} \delta^{-1}\left(\frac{(N K)^{1 / 4}}{L}\right)^{1 / 2}\left(\frac{\delta^{3 / 4} L^{9 / 8}}{(N K)^{1 / 4} L^{1 / 2}}\right)^{1 / 2} \\
& \ll T^{2 \kappa-1 / 8+\varepsilon}+T^{2 \kappa-1 / 2+\varepsilon} \delta^{-5 / 8} L^{-3 / 16} \\
& \ll T^{2 \kappa-1 / 8+\varepsilon}+T^{2 \kappa-1 / 2+\varepsilon} T^{5 / 16} L^{-3 / 16} \ll T^{2 \kappa-1 / 8+\varepsilon} .
\end{aligned}
$$

Case 2 If $\delta \ll L^{-3 / 2}$, then $\delta^{3 / 4} L^{9 / 8} \ll 1$. By Lemma 2.3, we have

$$
\delta \gg|\eta| \gg(n m k \ell)^{-1 / 2} \max (n, m, k, \ell)^{-3 / 2} \asymp(N K)^{-1 / 2} L^{-5 / 2} .
$$

Therefore, we obtain (recall $\delta \gg T^{-1 / 2}$ )

$$
\begin{aligned}
\mathcal{G}_{2} & \ll T^{2 \kappa-1 / 8+\varepsilon}+T^{2 \kappa-1 / 2+\varepsilon} \delta^{-1} \cdot \min \left(\frac{(N K)^{1 / 4}}{L}, \frac{1}{(N K)^{1 / 4} L^{1 / 2}}\right) \\
& \ll T^{2 \kappa-1 / 8+\varepsilon}+T^{2 \kappa-1 / 2+\varepsilon} \delta^{-1}\left(\frac{(N K)^{1 / 4}}{L}\right)^{1 / 4}\left(\frac{1}{(N K)^{1 / 4} L^{1 / 2}}\right)^{3 / 4} \\
& \ll T^{2 \kappa-1 / 8+\varepsilon}+T^{2 \kappa-1 / 2+\varepsilon} \delta^{-1}(N K)^{-1 / 8} L^{-5 / 8} \\
& \ll T^{2 \kappa-1 / 8+\varepsilon}+T^{2 \kappa-1 / 2+\varepsilon} \delta^{-1} \delta^{1 / 4} \\
& \ll T^{2 \kappa-1 / 8+\varepsilon}+T^{2 \kappa-1 / 2+\varepsilon} T^{3 / 8} \ll T^{2 \kappa-1 / 8+\varepsilon} .
\end{aligned}
$$

Combining (3.16) and (3.17), we get

$$
\mathcal{G}_{2} \ll T^{2 \kappa-1 / 8+\varepsilon} \text {. }
$$


For $\mathcal{G}_{3}$, by a splitting argument and Lemma 2.5 again, we get

$$
\begin{aligned}
\mathcal{G}_{3} & \ll \frac{T^{2 \kappa-1 / 2+\varepsilon}}{(N M K L)^{3 / 4} \delta} \times \sum_{\substack{\delta<|\eta| \leqslant 2 \delta \\
\delta \gg 1}} 1 \\
& \ll \frac{T^{2 \kappa-1 / 2+\varepsilon}}{(N M K L)^{3 / 4} \delta} \cdot \delta L^{1 / 2} N M K \ll T^{2 \kappa-1 / 2+\varepsilon}(N K)^{1 / 4} \\
& \ll T^{2 \kappa-1 / 2+\varepsilon} y^{1 / 2} \ll T^{2 \kappa-1 / 8+\varepsilon} .
\end{aligned}
$$

Comnining (3.7), (3.8), (3.13), (3.18) and (3.19), we get

$$
\int_{T}^{2 T} S_{2}(x) \mathrm{d} x \ll T^{2 \kappa-1 / 8+\varepsilon} .
$$

In the same way, we can prove that

$$
\int_{T}^{2 T} S_{3}(x) \mathrm{d} x \ll T^{2 \kappa-1 / 8+\varepsilon} .
$$

From (3.3)-(3.6), (3.20) and (3.21), we get

$$
\int_{T}^{2 T} A^{4}(x) \mathrm{d} x=\frac{3}{32 \pi^{4}} s_{4 ; 2}(\tilde{a}) \int_{T}^{2 T} x^{2 \kappa-1} \mathrm{~d} x+O\left(T^{2 \kappa-1 / 8+\varepsilon}\right),
$$

which implies Theorem 1.1 immediately.

\section{Acknowledgement}

The authors would like to express the most and the greatest sincere gratitude to Professor Wenguang Zhai for his valuable advice and constant encouragement.

\section{References}

[1] Y. C. Cai, On the third and fourth power moments of Fourier coefficients of cusp forms, Acta Math. Sinica (N.S.), 13 (4) (1997) 443-452.

[2] P. Deligne, La conjecture de Weil. I, Publ. Math. Inst. Hautes Études Sci., 43 (1) (1974) 273-307.

[3] A. Ivić, Large values of certain number-theoretic error terms, Acta Arith., 56 (2) (1990) 135-159.

[4] A. Ivić and P. Sargos, On the higher power moments of the error term in the divisor problem, Illinois J. Math., 51 (2) (2007) 353-377. 
[5] H. Joris, $\Omega$-Sätze für gewisse multiplikative arithmetische Funktionen, Comment. Math. Helv., 48 (1) (1973) 409-435.

[6] M. Jutila, Riemann's zeta-function and the divisor problem, Ark. Mat., 21 (1) (1983) 75-96.

[7] K. L. Kong, Some mean value theorems for certain error terms in analytic number theory, Master degree thesis, The University of Hong Kong (2014) .

[8] O. Robert and P. Sargos, Three-dimensional exponential sums with monomials, J. Reine Angew. Math., 591 (2006) 1-20.

[9] K. M. Tsang, Higher-power moments of $\Delta(x), E(t)$ and $P(x)$, Proc. London Math. Soc., 65 (3) (1992) 65-84.

[10] W. G. Zhai, On higher-power moments of $\Delta(x)$, Acta Arith., 112 (2004) 367-395.

[11] W. G. Zhai, On higher-power moments of $\Delta(x)(I I)$, Acta Arith., 114 (2004) 35-54.

[12] W. G. Zhai, On higher-power moments of $\Delta(x)(I I I)$, Acta Arith., 118 (2005) 263-281. 